\title{
Vulnerability to Depression: Cognitive Reactivity and Parental Bonding in High-Risk Individuals
}

\author{
Rick E. Ingram \\ Suuthern Methodist University
}

\author{
Jennifer Ritter \\ Arizona State University
}

\begin{abstract}
Although various conceptual proposals have suggested that disruptions in childhood bonding processes may be linked to the origins of these cognitive structures, little research has tested these proposals. This study assessed the information processing of vulnerable individuals and its relationship to childhood bonding. Formerly depressed (vulnerable) and never depressed (nonvulnerable) individuals participated in a mood induction task followed by an attentional allocation task. Results indicated that vulnerable individuals uniquely diverted attention toward negative stimuli when they were in a negative mood. Furthermore, level of maternal caring was found to be associated with performance on this task for vulnerable individuals in this mood state. These data support the idea that cognitive variables form a pathway between troublesome parental-child/adolescent interactions and depression.
\end{abstract}

Diathesis-stress models of depression suggest that depressogenic cognitive structures are dormant until activated by stressful events (e.g., Beck, 1967; Ingram, 1984; Ingram, Miranda, \& Segal, 1998: Teasdale, 1983). Segal and Shaw (1986) have described these structures as "latent but reactive" and argued that once triggered they initiate a pattern of negative self-referenced information processing that leads to depression. Segal and Ingram (1994) have noted that research support for these diathesis-stress models has grown substantially. In particular, converging lines of research have shown that vulnerable individuals process information dysfunctionally when encountering situations that activate depressive cognitive structures (e.g., Hedlund \& Rude, 1995; Ingram, Bernet, \& McLaughlin, 1994; Miranda, Gross, Persons, \& Hahn, 1998; Miranda, Persons, \& Byers, 1990; Teasdale \& Dent, 1987). Moreover, the processes suggested by diathesis-stress models have been shown to predict depression relapse in previously treated patients (Segal, Gemar, \& Williams, 1999) and to function in high-risk children as young as 8 years old (Taylor \& Ingram, 1999). Thus, depression-prone individuals appear to distinctly evidence negative cognitions, but these cognitions are not accessible until they experience an activating event.

Several theories spanning relatively diverse conceptual origins suggest that interactions with early caretakers provide the basis for negative information processing structures. For example, a variety of theories, including attachment theory (Bowlby, 1980), object relations theory (Baldwin, 1992; Westin, 1991), and several cog-

Rick E. Ingram, Department of Psychology, Southern Methodist University; Jennifer Ritter, Department of Psychology, Arizona State University.

Appreciation is expressed to Gerald Metalsky for a number of helpful comments on previous versions of this article, and to Robin Jarrett for her helpful comments on the interpretation of the results.

Correspondence concerning this article should be addressed to Rick E. Ingram, Department of Psychology, Southern Methodist University, Dallas, Texas 75275. Electronic mail may be sent to ringram@mail.smu.edu. nitive theories (Beck, 1967; Ingram et al., 1998; Segal, 1988), have suggested that caretakers who are unduly punitive, harsh, critical, or neglectful lead to both the development and linkage of affective structures and negative cognitive schemas. Even though they vary in theoretical details, a central conceptual theme that occurs throughout these proposals is that disruptions in the basic bonding processes between children and their caretakers produce vulnerability structures (Ingram et al., 1998). Moreover, a substantial body of work argues that depression in adulthood is related to the development of these variables in childhood (Bemporad \& Romano, 1992), suggesting that these vulnerability structures form the core of depression that occurs throughout the life span.

One area in which interactions with carly caretakers may be disrupted, and thus result in cognitive vulnerability, is in the relationship between depressed mothers and their children. For example, Garber and Robinson (1997) have shown that the children of depressed mothers evidence more self-criticism than do children whose mothers are not depressed. Likewise, Jaenicki et al. (1987) found that the offspring of depressed mothers had a more negative attributional style and demonstrated less positive information processing than did the offispring of nondepressed mothers. Recent data have also suggested that the children of depressed mothers evidence cognitive schemas that are reactive to events that produce negative emotion (Taylor \& Ingram, 1999). These data clearly suggest that bonding disruptions may result in cognitive vulnerability.

Although there are a variety of ways to conceptualize disruptions in basic bonding processes, Parker (1979) has offered a useful perspective that focuses specifically on lack of care and overprotection. Based on data from a variety of sources, Parker (Parker, 1983b, 1989, 1994; Parker, Tupling, \& Brown, 1979) has argued that these dimensions capture not only the core characteristics of most parental behaviors but also underlie interpersonal interactions with people in general. Thus, to the extent that parents do not provide an appropriate level of care, or are overprotective, basic bonding processes will be disrupted and individuals will 
become vulnerable to psychopathology. Indeed, several reviews have shown that disruptions in early interactions with parents, particularly those related to deficits in care, are linked to a greater likelihood of experiencing depression (e.g., Blatt \& Homann, 1992; Burbach \& Borduin, 1986; Gerlsma, Emmelkamp, \& Arrindell, 1990). Moreover, these reviews have documented consistent relationships between depression and scores on the Parental Bonding Instrument (PBI; Parker et al., 1979), a measure developed specifically to assess the care and protection dimensions of parental bonding (Parker, 1981, 1983a). Other research that has examined similar bonding dimensions, but which has not used the PBI, has also shown that reports of bonding disruptions are related to the type of self-regulation patterns (Manian, Strauman, \& Denney, 1998) that may be linked to affective distress.

Disruptions in the appropriate level of parental protectiveness, and particularly in level of parental care, thus seem likely candidates for producing the negative self-structures that are activated in vulnerable individuals when negative mood-producing events occur. However, despite the growing body of research that suggests that vulnerable individuals possess reactive cognitive selfstructures (e.g., Segal \& Ingram, 1994), little empirical research has assessed whether these proposed bonding origins are in fact associated with such structures. Moreover, research has not assessed which particular aspects of parental bonding might be associated with reactive cognitive schemas. Hence, little is known empirically about the bonding origins of reactive schemas in vulnerable individuals in general and, in particular, whether maternal or paternal factors, caring or overprotectiveness, or some combination of these dimensions are linked to reactive cognitive processing in depression vulnerability.

Accordingly, in the current study, we predicted an association between parental bonding and cognitive reactivity in depressionvulnerable individuals when in a negative mood, although note that previous data do not allow for specific predictions regarding which particular aspects of parental bonding would be related to cognitive reactivity. In order to assess this relationship, vulnerable and nonvulnerable participants completed the PBI prior to the assessment of information processing. Vulnerability was operationalized according to the previous experience of a clinically significant episode of depression (Segal \& Ingram, 1994). In accordance with experimental challenge methods used in numerous tests of diathesis-stress models in both psychological and biological research (Hollon, 1992; Segal \& Ingram, 1994), participants received a negative mood induction that was intended to model the emotion-producing effects of negative events.

Following from previous research, we assessed cognitive structure activation and consequent processing reactivity by examining attentional allocation on a dichotic listening task (Ingram et al., 1994). The principle underlying this measure is that negative schema activation should direct attention toward depressotypic information (see Neisser, 1967, 1976). The task requires participants to track the contents of a target message while a competing distractor message consisting of emotionally relevant information is also delivered. To the extent that the distractor information is pertinent to the individual's active cognitive processing structures, attention will be diverted from the target stimuli to the distractor stimuli and lead to an increased number of tracking errors for the target message (Lachman, Lachman, \& Butterfield, 1979).
Method

\section{Research Participants}

Research participants were recruited using a two-phase multiple gatc approach suggested by Kendall, Hollon, Beck, Hammen, and Ingram (1987). All participants were selected from a pool of approximately 1,200 individuals who participated in a mass screening session for their introductory psychology classes at San Diego State University. During this session, they completed measures of parental bonding, past depression, and current depressive symptoms. If participants indicated on a DSM-IV (Diagnostic and Statistical Manual of Mental Disorders, 4th ed.; American Psychiatric Association, 1994) criteria-based screening questionnaire that they had previously experienced at least five of the nine criteria for major depression for at least 2 weeks, they were considered to have experienced a past episode of depression. Never depressed control participants were defined according to reporting having never experienced five of the nine symptoms for a 2-week period. To ensure that the sample was not currently depressed, participants were selected only if they scored 7 or less on the Beck Depression Inventory (approximately the mean of the mass screening sample).

During the experimental phase, we used a screening procedure to determine the final sample. This second phase consisted of administration of portions of the Structured Clinical Interview for DSM-IV (SCID) at the conclusion of the experiment. ${ }^{.}$For formerly depressed individuals, this clinical interview was intended to confirm that their previous depressive episode met $D S M-I V$ criteria. If individuals who had indicated an episode of depression during the mass screening did not meet these additional criteria, their data were excluded from the study. Participants' data were also excluded if their depressive episode was part of a bipolar disorder. Data for never depressed participants were excluded if they met four or more depression criteria on the SCID. The BDI was readministered at the time of the experiment and participants were excluded if their BDI scores were above 7 .

The final sample consisted of (a) 35 formerly depressed individuals (26 women and 9 men) who had met criteria for a past episode of depression (on both the screening measure and the SCID) and who had indicated during both the mass screening and the actual experiment that they were not currently depressed, and (b) 38 never depressed individuals ( 21 women and $17 \mathrm{men}$ ) who were not currently depressed and who did not meet criteria for a past episode of depression on either occasion (on either the screening measure or the SCID). The predominance of women in the formerly depressed group is consistent with sex differences in the incidence of depression. Ethnicity was predominantly Caucasian and was equally distributed across each group. BDI and PBI data for these two groups are presented in Table 1, and PBI intercorrelations are presented in Table 2.

Prior to the experiment, each participant was randomly assigned to either the mood condition ( 16 formerly depressed and 23 never depressed participants) or the control condition (19 formerly depressed and 15 never depressed participants). The number in each group varied somewhat because of participants who did not show up for the experiment, individuals selected as formerly depressed who participated in the experiment but who were excluded from analyses because they did not meet diagnostic criteria, and participants who were excluded because they scored in the depressed range of the BDI on arrival for the experiment. There were no significant differences on any of the screening variables between participants who did and did not actually participate in the experiment.

\footnotetext{
' Because the SCID is a fairly lengthy assessment, it was given at the end of the experiment so as to both minimize sensitization to the nature of the study and minimize fatigue during the experimental parts of the study.
} 
Table 1

Means for BDI and Maternal and Paternal PBI and for Formerly and Never Depressed Groups

\begin{tabular}{lcc}
\hline Variable & Formerly depressed & Never depressed \\
\hline BDI & & \\
$M$ & 3.49 & 2.13 \\
$S D$ & 1.81 & 2.51 \\
PBI Maternal & & \\
Care & & \\
$M$ & 26.40 & 29.30 \\
$S D$ & 8.45 & 7.14 \\
Overprotection & & 12.82 \\
$M$ & 14.71 & 5.34 \\
$S D$ & 7.90 & \\
PBI Paternal & & 25.12 \\
Care & & 8.79 \\
$M$ & 23.51 & \\
$S D$ & 8.28 & 12.30 \\
Overprotection & & 6.75 \\
$M$ & 15.29 & \\
$S D$ & 8.20 & \\
\hline
\end{tabular}

Note. $\mathrm{PBI}=$ Parental Bonding Instrument; $\mathrm{BDI}=$ Beck Depression Inventory.

\section{Measures}

Parental bonding. To assess parental bonding, we administered the Parental Bonding Instrument (PBI; Parker et al, 1979) during the mass screening session at least 2 weeks prior to the experimental session. The PBI is a self-report questionnaire that measures the recall of parenting attitudes and behaviors (i.e., caring and protection) in an individual's first 16 years. Differential parenting styles are based on how respondents rate each parent on a Caring subscale (comprising 12 items) and a Protection subscale (comprising 13 items).

Numerous psychometric studies have established that the PBI possesses adequate reliability and validity (Parker, 1989, 1990). For example, in a review of the psychometric properties of the PBI, Parker (1989) reported internal consistencies ranging from .74 to .95 , with a median alpha coefficient of .89 and a mean alpha coefficient of .88 . He also reports test-retest reliability data from different countries (e.g., the United States and Australia), across a wide range of populations (e.g., community samples, depressed samples, and schizophrenic samples) and at various intervals (3-34 weeks). Test-retest reliabilities have been found to range between .58 (for a schizophrenic sample) and .96 (for an outpatient depressed sample tested before and after remission). The median test-retest reliability reported by Parker (1989) was .80 and the mean was .82 . Additionally, Wilhelm and Parker (1990) have reported reliability data over a 10-year period that ranges between .56 and .72 , which they interpreted as indicating considerable stability in the PBI over an extended period of time. PBI scores have also been shown to relate to actual parental behavior (Parker, 1984) and to correspond to parents' own perceptions and to that of siblings' ratings of parents (Parker, 1981; Parker et al., 1979). Data further show that the PBI assesses the presence of a risk factor for depression (Parker, 1983a), is consistently related to depression (Gerlsina, Emmelkamp, \& Arrindell, 1990; Parker, 1979, 1993, 1994), remains stable after depression remits (Gotlib, Mount. Cordy, \& Whiffen, 1988), and predicts the onset of depression (Gotlib, Whiffen, Wallace, \& Mount, 1991). In line with research showing the validity of the PBI, studies have failed to find that PBI scores are unduly influenced by the respondent's current emotional or depressive state (Brewin, Andrews, Gotlib, 1993).

Depressive symptomatolagy. We used the Beck Depression Inventory (BDI; Beck, 1967) to assess current depressive symptomatology. The BDI is a 21 -item self-report measure of a range of depressive symptoms. Each item is answered on a 0 to 3 scale with total scores ranging from 0 to 63 . Beck, Steer, and Garbin (1988) examined research assessing the psychometric properties of the BDI over a period of 25 years and have shown that this measure consistently demonstrates strong reliability and validity.

Negative affect. To assess the level of negative affect within the experimental session, we administered the Depression, Anxiety, and Hostility scales of the Multiple Affect Adjective Checklist (MAACL; Zuckerman \& Lubin, 1965) to participants. The combined MAACL scale contains 132 items; participants are instructed to check each item that describes how they felt at that particular moment. Participants received a point for each negative adjective they endorsed and a point for each positive adjective not endorsed. Like the BDI, the psychometric properties of the MAACL have been evaluated in numerous studies that have demonstrated that this measure is both reliable and valid (Lubin, Zuckerman, \& Woodward, 1985).

Previous depression screening. We adapted the screening measure used during the mass testing session from DSM-IV criteria for a major depressive episode and presented it to participants in a true/false format. This measure presented participants with a description of each of the nine depressive symptoms listed in $D S M-I V$ and were asked to answer true or false for each individual symptom according to whether or not they had experienced the symptom for at least a 2-week period. As noted in the Participants section, this measure constituted only a screening measure; the SCID was administered to confirm the diagnosis of a previous episode of depression.

Attentional allocation. The dichotic listening task in the present study was similar to that used by Ingram et al. (1994). In this task, participants listened to information presented to different ears. In one ear, they heard a continuous, affectively neutral story. They were asked to shadow this story by repeating it word for word aloud into a microphone. While participants shadowed this story, they were also presented with a series of distractor words, one at a time at approximately 10-s intervals, in their other ear. They were told to ignore any information in the other ear and attend only to the story they were to track. So that participants could clearly distinguish the story from the distractor words, the story was recorded with a female voice and the distractor words were recorded by a male voice at the same volume. Participants were allowed to adjust the overall volume to a level that they found comfortable.

Table 2

PBI Intercorrelations for the Total Sample and for Each Vulnerability Group

\begin{tabular}{|c|c|c|c|c|}
\hline \multirow[b]{2}{*}{ Group } & \multicolumn{4}{|c|}{ PBI scales } \\
\hline & $\mathrm{FC}$ & FP & $\mathrm{MC}$ & MP \\
\hline \multicolumn{5}{|l|}{ Father care (FC) } \\
\hline Total sample & - & & & \\
\hline Vulnerable & - & & & \\
\hline Nonvulnerable & - & & & \\
\hline \multicolumn{5}{|c|}{ Father overprotection (FP) } \\
\hline Total sample & $-.29 *$ & - & & \\
\hline Vulnerable & -.18 & $\ldots$ & & \\
\hline Nonvulnerable & $-.51^{*}$ & - & & \\
\hline \multicolumn{5}{|l|}{ Mother care (MC) } \\
\hline Total sample & $.52 *$ & $-.36^{*}$ & - & \\
\hline Vulnerable & $.51^{*}$ & -.26 & - & \\
\hline Nonvulnerable & $.53 *$ & $-.44 *$ & - & \\
\hline \multicolumn{5}{|c|}{ Mother overprotection (MP) } \\
\hline Total sample & $-.29 *$ & $.42 *$ & $-.54 *$ & - \\
\hline Vulnerable & -.27 & $-.44 *$ & $-.55^{*}$ & - \\
\hline Nonvulnerable & -.31 & $.35 \%$ & $-.50^{*}$ & - \\
\hline
\end{tabular}

Note. $\mathrm{PBI}=$ Parental Bonding Instrument.

$* p<.05$. 
Distractor words were ordered in blocks or sets of affectively similar words. Three blocks were created that consisted of a (a) nine negative adjectives (e.g., sad, alone, unhappy), (b) nine positive adjectives (e.g., cheerful, happy, amused), and (c) nine neutral nouns (e.g., state, outside, chair). A fourth block consisted of a set of 13 neutral words that were always presented first and was considered to serve as a performance baseline to account for individual differences in attentional tracking ability. For the task used in the present study, following this baseline, we presented the negative, positive, and neutral distractor blocks. Blocks were presented in a random order, thus insuring that study participants received different orders of the distractor blocks. Prior to beginning the baseline, we gave all participants a short practice session to ensure that they understood the task. Thus, after this practice session, in one ear, we presented participants with the baseline words, and then randomly ordered blocks of negative, positive, and neutral adjectives while they listened to and attempted to verbally track a story in the other ear.

Each participant's performance was tape-recorded for later scoring Errors that occurred for the tracking story at any point during the presentation of these three blocks of adjectives constituted the dependent variable (i.e., errors that occurred in story tracking at any point during the presentation of the negative distractor word block were referred to as negative errors, errors that occurred at any point during the block of positive distractor words were referred to as positive errors, and errors during the presentation of neutral words were referred to as neutral errors). In this way, the task gauges the extent of attention that was directed away from the target task and toward information that was negative, positive, or neutral, respectively.

Scoring of story-tracking errors was done independently by two research assistants who were unaware of vulnerability status and mond condition. Reliability of .89 between the two scores was quite acceptable. Scores were averaged between the two raters and the mean score was used in all analyses. Five possible types of errors during the tracking of the story were scored: missed words, incorrectly stated words, intrusions of other words (such as speaking the distractor word), dysfluencies such as stuttering, and extra words such as repeating a word that had already been said. Errors in these five categories were totaled for each block of distractor words and thus a total error score for each of the three blocks was used in the analyses.

\section{Procedure}

As noted, we recruited participants through a mass testing procedure of introductory psychology students. A minimum of 2 weeks after the mass screening, we contacted participants who met initial study criteria and asked if they would be interested in participating in the study. On arrival, participants were informed that they would be participating in an experiment to investigate cognitive processes in persons who had and had not previously experienced depression. After giving informed consent, each participant was tested individually. Participants were first readministered the BDI and then participated in either the mood induction or the control condition. They did so by wearing headphones that presented the instructions for the task as well as the mood or control condition stimuli. Immediately following this procedure, participants completed the MAACL and then began the dichotic listening task. In this task they were instructed to shadow or repeat the message word for word and to ignore any other words that they heard in the other ear. Following completion of this task, participants were administered the SCID and were then debriefed.

The mood induction was a combination of two methods that were similar to those used by Ingram et al. (1994). Participants were asked to listen to sad music and were asked to think about a sad event in their lives. The music consisted of two selections from the soundtrack for the movie Field of Dreams. This music continued for approximately $8 \mathrm{~min}$. In the control condition, participants listened to a series of random sounds while they examined a children's storybook. This condition was intended to have no effect of participants' mood levels.
Results

\section{PBI Scores, Initial Depression Levels, and Mood Induction}

Although each of the PBI means for the vulnerable group were somewhat higher than those for the nonvulnerable group, none of these differences were statistically significant. As noted, intercorrelations between each of the PBI subscales for the sample as a whole, and for each vulnerability group separately, are presented in Table 2 . The only clear discrepancy between the groups was that whereas father care and father overprotection were significantly related for nonvulnerable participants, there was little correlation between these measures for vulnerable participants. ${ }^{2}$

Even though only those participants scoring in the nondepressed range of the BDI were selected for participation, it was still necessary to examine potential group differences in $\mathrm{BDl}$ scores to ensure that one group was not at the higher end of the nondepressed range and the other group was at the lower or middle ranges, and to also ensure that the vulnerable group did not experience increased depressive symptoms in the time intervening between selection and participation in the experiment. Therefore, we conducted a $2 \times 2$ (Vulnerability Status $\times$ Mood Condition) analysis of variance (ANOVA) on BDI scores. This analysis found only a significant main effect for vulnerability status, $F(1$, $69)=6.03, p=.02$, indicating that the vulnerable participants had somewhat higher BDI scores than nonvulnerable participants, although note that all participants were well within the nondepressed range on the $\mathrm{BDI}$.

To assess the efficacy of the mood induction, we analyzed the overall MAACL, composed of all three subscales, in a $2 \times 2$ analysis of covariance (ANCOVA) controlling for differences in BDI scores. This analysis indicated that although differences in mood were not exceptionally large, participants in the mood induction condition scored significantly higher $(M=22.31$, $S D=9.69)$ than did control condition participants $(M=16.82$, $S D=8.94), F(1,68)=6.55, p<.02$. No other differences approached significance. To assess the specificity of affective responding, each MAACL subscale was then examined in a ANCOVA that controlled for BDI scores and the remaining MAACL subscales. This analysis indicated that the main effect for mood induction held up only for the MAACL Depression subscale, $F(1,68)=4.85, p<.03$ ( $p s>.39$ for Anxiety and Hostility).

\section{Effects of Mood Induction and Depressive History on Attentional Allocation}

We conducted a $2 \times 2$ between-subjects multivariate analysis of covariance (MANCOVA) on positive, negative, and neutral dichotic listening errors. In this MANCOVA we used baseline scores as a covariate to control for individual differences in tracking

\footnotetext{
${ }^{2}$ Apparent discrepancies in significance levels reflect differing sample sizes. For example, whereas -.29 is significant for the total sample $(N=$ 73), when the sample is divided according to vulnerability groups the larger correlation of -.31 is not significant.
} 


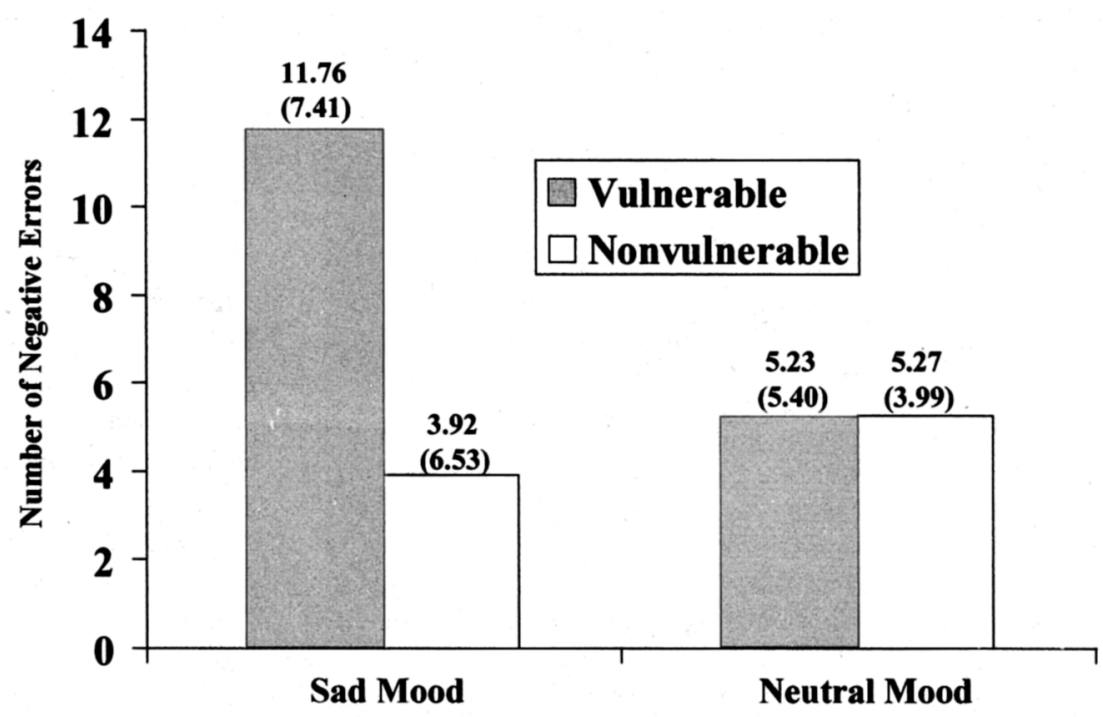

Figure 1. Adjusted number of negative errors as a function of mood condition and vulnerability to depression. Means are on top and standard deviations are in parentheses.

ability (Ingram et al., 1994). ${ }^{3}$ This analysis indicated a significant Mood Induction $\times$ Vulnerability Status interaction, $F(3$, 66) $=3.56, p<.02$. To determine the specific nature of this interaction. we conducted follow-up univariate ANCOVAs separately on each of the error types. No effects approached significance for either positive or neutral errors (e.g., $p s>.30$ ). However, we found several significant effects for negative errors, specifically, a main effect for vulnerability status, $F(1$, $68)=12.70 . p<.001$, and a trend for mood induction condition, $F(1,68)=3.19, p=.079$. These were qualified by a significant interaction, $F(1,68)=7.58, p<.008$. The adjusted means comprising this interaction are presented in Figure 1.

Bonferroni-corrected tests of simple main effects (critical value per comparison $p=.0125$ ) revealed a simple main effect of mood induction, $F(1,68)=7.10, p=.01$, which indicated that formerly depressed participants in the sad mood condition made significantly more negative errors than did formerly depressed participants in the neutral mood condition. We also found a significant simple main effect of depression history in the sad mood condition, $F(1,68)=18.97, p<.001$, with formerly depressed individuals again making more negative errors than individuals who had never experienced a depressive episode. In the neutral mood condition we found no difference between formerly depressed participants and never depressed participants $(p=.63$ ), nor did mood affect participants who had no history of depression $(p=.34)$.

\section{Relationship Between Parental Bonding, Mood, and Depression History on Attention Allocation}

Because the number of study participants was not sufficient to permit a stepwise hierarchical regression analysis, to examine the prediction that there would be an association between parental bonding and cognitive reactivity in depression-vulnerable individuals, we calculated correlation coefficients for both positive and negative errors on the dichotic listening task and the four PBI subscales. These correlations were conducted separately for indi- viduals in each of the experimental conditions. Correlation coefficients are presented as Table 3. Among never depressed individuals, none of the PBI subscales were significantly associated with negative error rates or positive error rates in either the sad mood condition or the neutral mood condition. In contrast, for formerly depressed individuals in the sad mood condition, maternal care was significantly ncgalively correlated with the number of negative errors made on the dichotic listening task $(r=-.57, p<.02)$, suggesting that decreased maternal care was associated with schema-mediated processing of negative information. The correlations between maternal care and positive errors in the sad mood condition ( -.38 ), and in the neutral mood condition between negative errors and maternal overprotectiveness $(r=-.39)$ and paternal overprotectiveness $(r=-.39)$, were only marginally significant $(p s<.12)$.

In light of the significant association between maternal caring and negative errors in the mood induction condition, we conducted a three-way ANCOVA assessing bonding (higher vs. lower matemal caring), depression history, and mood induction to better understand how maternal caring influenced the relationship between history of depression and mood induction on attention allocation. For purposes of this ANCOVA, we dichotomized scores on the maternal caring subscale of the PBI, using a median split procedure $(M d n=31)$. A total of 35 research participants were classified as having received lower levels of maternal care, and 38 participants were classificd as having received higher levels of maternal care. As before, we used baseline dichotic listening scores as the covariate.

\footnotetext{
${ }^{3}$ All analyses involving dichotic task performance were aiso conducted. with BDI scores as a covariate. In no case did inclusion of this variable alter the pattern of results, most likely because the difference, although statistically significant, was quite small in magnitude (less than 2 points) and because all participants were well within the nondepressed range.
} 
Table 3

Correlations Between Attention Allocation Performance and Parental Bonding Scores as a Function of Vulnerability to Depression and Mood Condition

\begin{tabular}{|c|c|c|c|c|c|c|c|c|}
\hline \multirow[b]{2}{*}{ Condition } & \multicolumn{4}{|c|}{ Vulnerable } & \multicolumn{4}{|c|}{ Nonvuinerable } \\
\hline & MC & MP & $\mathrm{FC}$ & FP & MC & MP & $\mathrm{FC}$ & FP \\
\hline \multicolumn{9}{|l|}{ Negative mood } \\
\hline Neg. stimuli errors & $-.57 *$ & .07 & -.13 & .16 & .11 & -.08 & .04 & .09 \\
\hline Pos. stimuli errors & -.38 & .09 & -.05 & .06 & -.04 & -.11 & .02 & .15 \\
\hline \multicolumn{9}{|l|}{ Neutral mood } \\
\hline Neg. stimuli errors & -.05 & -.39 & -.16 & -.39 & .06 & .04 & -.04 & -.11 \\
\hline Pos. stimuli errors & -.18 & .26 & -.27 & .29 & -.09 & .17 & .09 & .06 \\
\hline
\end{tabular}

Note. $\quad \mathrm{MC}=$ maternal caring; $\mathrm{MP}=$ maternal overprotection; $\mathrm{FC}=$ father caring; $\mathrm{FP}=$ father overprotection; Neg. $=$ negative; Pos. $=$ positive.

$* p<.05$.

This analysis revealed a near significant three-way interaction between level of maternal caring, depression history, and mood induction procedure on attention allocation, $F(1,64)=3.73, p=$ .058. Although not quite significant, several factors suggest that it is reasonable to further explore this interaction. For example, using a covariate and dividing the sample by another variable to create a three-way design diminishes the power to detect a significant higher order interaction. Moreover, given that the purpose of this analysis was to help better understand the significant correlation between maternal care and negative errors, it made sense to examine the simple interaction of bonding and depressive history within each mood induction procedure.

No differences in the neutral mood condition approached significance (all $p s>.20$ ). However, further follow-up testing in the mood condition (Bonferroni-corrected critical value of $p=.0125$ ) revealed a significant simple main effect of caring among individuals with a previous history of depression, $F(1,64)=12.15, p<$ .001 , such that individuals with a depression history and lower maternal caring evidenced more negative errors on the dichotic listening task (approximately twice as many) than individuals with a depression history and higher maternal caring. Level of maternal caring was not related to dichotic listening errors in individuals without a history of depression ( $p=.74$ ). Similarly, another effect for depression history was revealed among individuals who received lower maternal caring, $F(1,64)=21.29, p<.001$. This result indicated that, for those who reported lower levels of care, individuals with a history of depression made more negative errors than did individuals without a history of depression. The effect of depression history among individuals higher in maternal caring was not significant ( $p=.14$ ). The adjusted means comprising this interaction are depicted in Figure 2.

\section{Discussion}

Results of the current study indicated that when experiencing a sad mood, vulnerable individuals were more distracted by negative

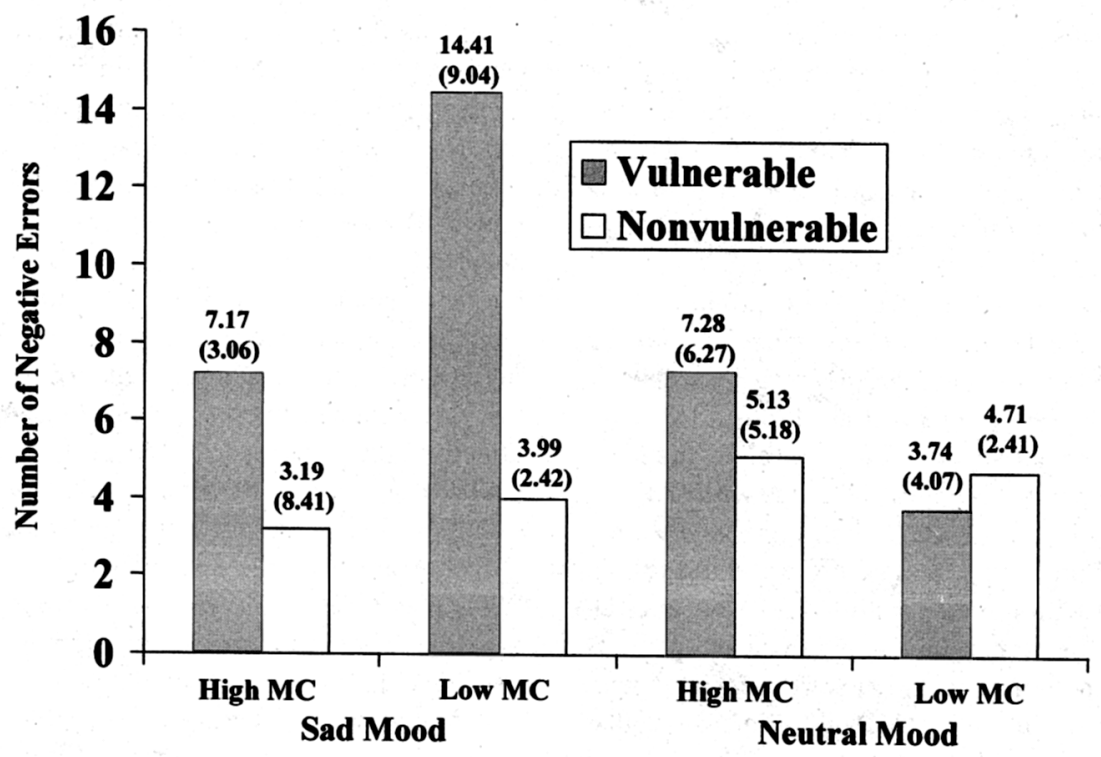

Figure 2. Adjusted number of negative errors as a function of mood condition, vulnerability to depression, and level of maternal caring (MC). Means are on top and standard deviations are in parentheses. 
stimuli than were nonvulnerable individuals. These participants were also more distracted than vulnerable individuals who did not experience a sad mood state. These findings are consistent with data reported by Ingram et al. (1994) regarding negative information processing on this cognitive task and, more broadly, are consistent with diathesis-stress models of depression that predict schema reactivity when triggering events occur (Ingram et al., 1998; Segal \& Shaw, 1986). The current study also found that vulnerable and nonvulnerable individuals did not differ in the attention they accorded to negative stimuli when they were not experiencing a negative mood state. These data closely parallel previous findings that have failed to find evidence of negative cognitive processing after depression remits. However, along with data from other priming studies (Segal \& Ingram, 1994), the current findings suggest that cognition as a potential causative agent must be assessed within the context of the activating features of affectively linked events.

An important goal of this study was to assess possible relationships between parental bonding experiences and depressotypic cognitive processing under conditions of negative emotion. The most consistent finding in this regard was that deficits in maternal care, assessed several weeks prior to the experiment, were related to primed negative information processing in depression-prone individuals during the experiment. In particular, the correlational analyses indicated that lower levels of maternal caring were associated with enhanced attention to negative stimuli for vulnerable individuals who were in a sad mood. Additionally, the follow-up procedure separating participants into higher and lower maternal care groups suggested that individuals with a history of depression and lower levels of maternal caring accorded more attention to negative stimuli when in a negative mood state than did individuals with a history of depression and higher levels of maternal care when in a negative mood. Taken together, these analyses indicate that the more neglect or rejection that vulnerable individuals perceived on the part of their mothers, the more likely they were to evidence depressive information processing when encountering a negative mood. Note, however, that because the three-way interaction was not quite statistically significant $(p=.058)$, replication of these results will be important. Nevertheless, when these results are considered along with the correlational analyses, the current study suggests that one way that bonding is linked to depression in adulthood through the deleterious effects of low levels of maternal care on the formation and activation of negative cognitive schemas.

The current data are consistent with theoretical proposals that argue that disruptions in the bonding process are related to the creation of negative schemas that place people at risk for depression (Baldwin, 1992; Beck, 1967; Ingram et al., 1998). Moreover, these data suggest that deficits specifically in level of maternal care play a key role, a finding that is generally in line with Gerlsma et al's. (1990) meta-analytic review of the link between bonding and psychopathology. From a very different perspective, these results are also consistent with research reported by Manian et al. (1998), who found that the types of self-discrepancy patterns thought to be related to emotional regulation were associated with recollections of parenting warmth and rejection-dimensions that are quite similar to the Caring scale of the PBI. Although the measure Manian et al. (1998) used did not allow them to differentiate between maternal and parental characteristics, other studies have found that depression-related variables are specifically associated with deficits in maternal care. For instance, Alnaes and Torgersen (1990) reported that individuals with a history of depression had significantly lower maternal care scores than did individuals with anxiety or mixed depression and anxiety. Likewise, Parker (1979) found maternal care to be uniquely associated with depressive rather than anxious symptoms and also found that the kind of cognitive deficits that are frequently seen in depression are associated with a lack of maternal care. Additionally, Ingram, Overbey, and Fortier (2000) found that maternal care, but not other parenting dimensions, was associated with deficits in positive cognition and excesses in negative cognition. In accord with these studies, the current data broadly suggest that care is an important process in depression and further indicate that deficits specifically in maternal care may play a significant role in the development of depressogenic cognitive variables.

Although previous data as well as the current study suggest the importance of maternal care deficits in producing cognitive functioning that is linked to vulnerability to depression, theoretical models examining the long-term effects of child-parent bonding do not typically postulate why maternal care seems to be more strongly linked to this process than are other parenting variables (i.e., maternal overprotection, paternal care, and paternal overprotection). It will thus be important for models of cognitive vulnerability to depression to address the factors that may account for such specificity. One possibility in this regard is that the tendency to process information negatively in a depressed mood state may be traced to high levels of maternal depression. That is, deficits in maternal caring have been shown to be linked to maternal depression (Hammen, 1991). The current findings may therefore indicate that mothers who are depressed provide less care and consequently engender a tendency for their children to think negatively (Garber \& Robinson, 1997; Jaenicki et al., 1987), especially when these children experience sad mood states (Taylor \& Ingram, 1999). Although only speculative, deficits found in maternal care may thus represent a proxy of sorts for having a mother who experiences chronic negative affective states.

Despite the fact that models do not tend to examine the specificity of maternal care in producing cognitive vulnerability, in general, findings suggesting that maternal care is associated with dysfunctional cognition are broadly in line with evolutionary approaches to attachment and depression suggested by Bowlby (1980). Bowlby argued that humans are genetically predisposed to develop bonding processes with key caretakers and that if this bonding is sufficiently disrupted during development, then sadness, despair, and eventually depression will result. In this regard, Ingram et al. (1998) emphasized that cognitive structures are not the only neural networks that are developing as children mature; affective structures are similarly becoming both well differentiated and well connected to other structures. When neglect is experienced from such an important attachment figure as one's mother, not only are negative self-images produced but it seems quite likely that these images become strongly tied to the experience of negative affect. Thus, through extensive interactions with a mother perceived as uncaring, children may begin to internalize these matemal views and develop self-representations organized around seeing themselves as unworthy of attention and care (Batagos \& Leadbeater, 1994; Bowlby, 1980), which are linked to the experience of negative emotion. A neglectful mother may therefore 
propagate unfavorable views of the self and world that become intricately intertwined with negative affect in the vulnerable individual. When vulnerable individuals experience sadnessproducing events in the future, they will not only experience depressive affect but will also encounter the activation of cognitions about the self that reflect personal themes of unworthiness that have become deeply encoded in self-structures (Ingram et al., 1998).

Several recent perspectives have argued that a particular subset of high-risk individuals may be vulnerable for cognitive reasons (Abramson, Metalsky, \& Alloy, 1989; Ingram et al., 1998). The present results are generally consistent with these perspectives in that those individuals who evidenced the most disrupted bonding patterns, particularly those related to levels of maternal care, were also the most likely to process negative information when in a sad mood. Conversely, the fact that vulnerable individuals who reported better bonding made fewer errors than this group suggests the possibility that some people who experience a depressive episode are less likely to do so because of the availability of depressive cognitive structures; rather, these individuals may have been depressed for reasons other than reactive depressive cognitive structures. Biologically mediated depressions are an obvious possibility in this regard, although it is also possible that psychosocial factors other than cognitive variables, or extremely high levels of stress, precipitate depression for some people (Hollon, 1992).

In examining the possible implications of these results, it is also important to consider possible limitations. Although schema-based models guided this research, note that other theoretical explanations for the results cannot be ruled out (e.g., category accessibility constructs). Additionally, issues concerning generalizability must be addressed. The sample we used in this study was young, relatively well-educated, and had an early depression onset; some caution must therefore be exercised in any attempts to generalize these findings to individuals with a different set of demographic characteristics. Likewise, the sample size was fairly small and results should thus be interpreting accordingly. Finally, the present data cannot address issues of the specificity of parental bonding and subsequent cognitive vulnerability to depression. In particular, it is unclear whether the kinds of parental bonding patterns examined in this study are specific to depression or may also be related to other disorders (Downey \& Coyne, 1990).

In summary, the current study shows that affectively laden events uniquely provoke the activation of negative cognitive structures in individuals who are vulnerable to depression. These results parallel previous research showing that, under certain conditions, attentional resources are diverted in the direction of negative stimuli. The current data further suggest that such processes are moderated by low levels of maternal caring. When in a negative mood state, individuals with low maternal caring and a history of depression accord more attention to negative stimuli. The current data further suggest that such processes are moderated by low levels of maternal caring. When in a negative mood state, individuals with low maternal caring and a history of depression accord more attention to negative stimuli than do individuals with a history of depression and high maternal caring. Such data provide tantalizing clues on the origin of depressive cognitive processes, but future research must consider whether the variables reflecting these clues are indeed linked to the genesis of vulnerability in some people. Nevertheless, this research supports the idea that cognitive variables form a pathway between troublesome parentchild/adolescent interactions and depression.

\section{References}

Abramson, L. Y., Metalsky, G. 1., \& Alloy, L. B. (1989). Hopelessness depression: A theory-based subtype of depression. Psychological Review, 96, 358-372.

Alnaes, R., \& Torgersen, S. (1990). Parental representations in patients with major depression, anxiety disorder, and mixed conditions. Acta Psychiatrica Scandinavica, 81, 518-522.

American Psychiatric Association. (1994). Diagnostic and Statistical manual of mental disorders (4th ed.). Washington, DC: Author.

Baldwin, M. W. (1992). Relational schemas and the processing of social information. Psychological Bulletin, 112, 461-484.

Batgos, J., \& Leadbeater, B. J. (1994). Parental attachment, peer relations, and dysphoria in adolescence. In M. B. Sperling \& W. H. Berman (Eds.), Attachment in adults: Clinical and developmental perspectives (pp. 155-178). New York: Guilford Press.

Beck, A. T. (1967). Depression: Causes and treatment. Philadelphia: University of Pennsylvania Press.

Beck, A. T., Steer, R. A.. \& Garbin, M. G. (1988). Psychometric properties of the Beck Depression Inventory: Twenty-five years of evaluation. Clinical Psychology Review, 8, 77-100.

Bemporad, J. R., \& Romano, S. J. (1992). Childhood maltreatment and adult depression: A review of research. In D. Cicchetti \& S. L. Toth (Eds.), Developmental perspectives on depression (pp. 351-375). Rochester, NY: University of Rochester Press.

Blatt, S. J., \& Homann, E. (1992). Parent-child interaction in the etiology of dependent and self-critical depression. Clinical Psychology Review, 12, 47-91.

Bowlby, J. (1980). Attachment and loss: Vol. 3. Loss: Sadness and depression. New York: Basic Books.

Brewin, C. R., Andrews, B., \& Gotlib, I. (1993). Psychopathology and early experience: A reappraisal of retrospective reports. Psychological Bulletin, 113, 82-98.

Burbach, D. J., \& Borduin, C. M. (1986). Parent-child relations and the etiology of depression. Clinical Psychology Review, 6, 133-153.

Downey, G., \& Coyne, J. C. (1990). Children of depressed parents: An integrative review. Psychological Bulletin, 108, 50-75.

Garber, J., \& Robinson, N. S. (1997). Cognitive vulnerability in children at risk for depression. Cognition and Emotion, 11, 619-635.

Gerlsma, C., Emmelkamp, P. M. G., \& Arrindell, W. A. (1990). Anxiety, depression, and perception of early parenting: A meta-analysis. Clinical Psychology Review, 10, 251-277.

Gotlib, J. H., Mount, J. H., Cordy, N. I., \& Whiffen, V. E. (1988) Depression and perceptions of early parenting: A longitudinal investigation. British Journal of Psychiatry, 152, 24-27.

Gotlib, I. H., Whiffen, V. E., Wallace, P. M., \& Mount, J. H. (1991). Prospective investigation of postpartum depression: Factors involved in onset and recovery. Journal of Abnormal Psychology, 2, 122-132.

Hammen, C. (1991). Depression runs in families: The social context of risk and resilience in children of depressed mothers. New York: SpringerVerlag.

Hedlund, S., \& Rude, S. S. (1995). Evidence of latent depressive schemas in formerly depressed individuals. Journal of Abnormal Psychology. 104, 517-525.

Hollon, S. D. (1992). Cognitive models of depression from a psychobiological perspective. Psychological Inquiry, 3, 250-253.

Ingram, R. E. (1984). Toward an information processing analysis of depression. Cognitive Therapy and Research, 8, 443-478.

Ingram, R. E., Bernet, C. Z., \& McLaughlin, S. C. (1994). Attentional allocation processes in individuals at risk for depression. Cognitive Therapy and Research, 18, 317-332. 
Ingram, R. E., Miranda, J., \& Segal, Z. V. (1998). Cognitive vulnerability to depression. New York: Guilford Press.

Ingram, R. E., Overbey, T., \& Fortier, M. (in press). Individual differences in dysfunctional automatic thinking and parental bonding: Specificity of maternal care. Personality and Individual Differences.

Jaenicke, C., Hammen, C., Zupan, B., Hiroto, D., Gordon, D., Adrian, C., \& Burge, D. (1987). Cognitive vulnerability in children at risk for depression. Journal of Abnormal Child Psychology, 15, 559-572.

Kendall, P. C., Hollon, S. D., Beck, A. T., Hammen, C. L., \& Ingram, R. P. (1987). lssues and recommendations regarding use of the Beck Depression Inventory. Cognitive Therapy and Research, 11, 289-299.

Lachman, R., Lachman, J. L., \& Butterfield, E. C. (1979). Cognitive psychology and information processing. New York: Erlbaum.

Lubin, B., Zuckerman, M., \& Woodward, L. (1985). Bibliography for the Multiple Affect Adjective Checklist. San Diego, CA: Educational and Industrial Testing Service.

Manian, N., Strauman, T. J., \& Denney, N. (1998). Temperament, recalled parenting styles, and self-regulation: Testing the developmental postulates of self-discrepancy theory. Journal of Personality and Social Psychology, 75, 1321-1332.

Miranda, J., Gross, J. J., Persons, J. B., \& Hahn, J. (1998). Mood matters: Negative mood induction activates dysfunctional attitudes in women vulnerable to depression. Cognitive Therapy and Research, 22, 363-376.

Miranda, J., \& Persons, J. B. (1988). Dysfunctional attitudes are moodstate dependent. Journal of Abnormal Psychology, 97, 76-79.

Miranda, J., Persons, J. B., \& Byers, C. N. (1990). Endorsement of dysfunctional beliefs depends on current mood state. Journal of Abnormal Psychology, 99, 237-241.

Neisser, U. (1967). Cognitive psychology. New York: Appleton.

Neisser, U. (1976). Cognition and reality. San Francisco: Freeman.

Parker, G. (1979). Parental characteristics in relation to depressive disorders. British Journal of Psychiatry, 134, 138-147.

Parker, G. (1981). Parental reports of depressives: An investigation of several explanations. Journal of Affective Disorders, 3, 131-140.

Parker, G. (1983a). Parental "affectionless control" as an antecedent to adult depression: A risk factor delineated. Archives of General Psychiatry, 40, 956-960.

Parker, G. (1983b). Parental overprotection: A risk factor in psychosocial development. New York: Grune \& Stratton.

Parker, G. (1984). The measurement of pathogenic parental style and its relevance to psychiatric disorder. Social Psychiatry, 19, 75-81.

Parker, G. (1989). The parental bonding instrument: Psychometric properties reviewed. Psychiatric Developments, 4, 317-335.

Parker, G. (1990). The parental bonding instrument: A decade of research. Social Psychiatry and Psychiatric Epidemiology, 25, 281-282.
Parker, G. (1993). Parental rearing style: Examining for links with personality factor for depression. Social Psychiatry and Psychiatric Epidemiology, 28, 97-100.

Parker, G. (1994). Parental bonding and depressive disorders. In M. B. Sperling \& W. H. Berman (Eds.), Attachment in adults: Clinical and developmental perspectives (pp. 299-312). New York: Guilford Press.

Parker, G., Tupling, H., \& Brown, L. B. (1979). A parental bonding instrument. British Journal of Medical Psychology, 52, 1-10.

Segal, Z. V. (1988). Appraisal of the self-schema construct in cognitive models of depression. Psychological Bulletin, 103, 147-162.

Segal, Z. V., Gemar, M., \& Williams, S. (1999). Differential cognitive response to a mood challenge following successful cognitive therapy or pharmacotherapy for unipolar depression. Journal of Abnormal Psychology, $108,3-10$.

Segal, Z. V., \& Ingram, R. E. (1994). Mood priming and construct activation in tests of cognitive vulnerability to unipolar depression. Clinical Psychology Review, 14, 663-695.

Segal, Z. V., \& Shaw, B. F. (1986). Cognition in depression: A reappraisal of Coyne \& Gotlib's critique. Cognitive Therapy and Research, 10, 671-694.

Taylor, L., \& Ingram, R. E. (1999). Cognitive reactivity and depressotypic information processing in the children of depressed mothers. Journal of Abnormal Psychology, 108, 202-210.

Teasdale, J. D. (1983). Negative thinking in depression: Cause, effect, or reciprocal relationship? Advances in Behaviour Therapy and Research, 5, 3-25.

Teasdale, J. D., \& Dent, J. (1987). Cognitive vulnerability to depression: An investigation of two hypotheses. British Journal of Clinical Psychology, 26, 113-126.

Westin, D. (1991). Social cognition and object relations. Psychological Bulletin, 109, 429-455.

Wilhelm, K., \& Parker, G. (1990). Reliability of the Parental Bonding Instrument and Intimate Bond Measure scales. Australian and New Zealund Journal of Psychiatry, 24, 199-202.

Zuckerman, M., \& Lubin, B. (1965). Manual for the Multiple Affect Adjective Checklist. San Diego, CA: Educational and Industrial Testing Service.
Received October 1, 1997

Revision received January 12, 2000

Accepted January 15, 2000 